International Journal of Child, Youth, and Family Studies (2014): 5(4.2) 847-853

\title{
DOGWOOD ROOM ENTANGLEMENTS
}

\section{Adrienne Argent}

\begin{abstract}
This essay is a sample of pedagogical documentation from a toddler program. It seeks to highlight the unfolding processes and entanglements of ritual, materials, objects, and more. It discusses the concepts of intra-activity and assemblage as intricate networks of forces that bring complexity and generative potential to the lived and layered culture of children's spaces.
\end{abstract}

Keywords: early childhood education, curriculum, pedagogical documentation, assemblage, intra-activity, artistic processes

Adrienne Argent holds a bachelor's degree from the School of Child and Youth Care at the University of Victoria and is an early childhood educator at the Capilano University Children's Centre, 2055 Purcell Way, North Vancouver, British Columbia, Canada V7J 3H5.

E-mail: a.argent@hotmail.com

The Dogwood Room is located in a children's centre on a university campus. Within the children's centre the educators work with an atelierista (art studio teacher) and a pedagogista (pedagogical facilitator). Both of these roles are central to the Reggio Emilia teaching philosophy. The philosophical principles of the Reggio Emilia schools inspire us in our thinking. However, we seek not to imitate, but rather are steadfast in our efforts to speak and act with authenticity.

In the Dogwood Room, we are a collective that includes children (ages 18 months to 29 months), parents, and four educators. There are many identities and rhythms that are in constant circulation, sometimes blending together and at other times colliding. Materials and objects such as wood, paper, and clay are also active participants. We are in constant partnership with these objects as they become an extension of our voices and our bodies. These objects travel with us as 
we create, construct, and deconstruct ideas together. There is a distinct soundscape to the Dogwood Room that is difficult to describe because it is an ever-changing pulsation of expression. Together, we are in a constant state of negotiation, as our movements and affectations flow through the room, producing reverberations that can be both potent and delicate. To say that we play together is an unjust oversimplification: Rather we are in an ongoing process of becoming, no beginning or end-fluid-dynamic-generative-highly emotive. Our curriculum is lived out daily; it exists with(in) all of us. Clay, paper, materials, children, educators, objects, music and....and.... and, are all powerful forces and they bring forth movement, history, and multiple layers of meaning. In the Dogwood Room we understand curriculum as an entanglement of time, place, bodies, materials, and more. Curriculum is not situated outside the child, rather the two become enmeshed, (re)acting and transforming each other.

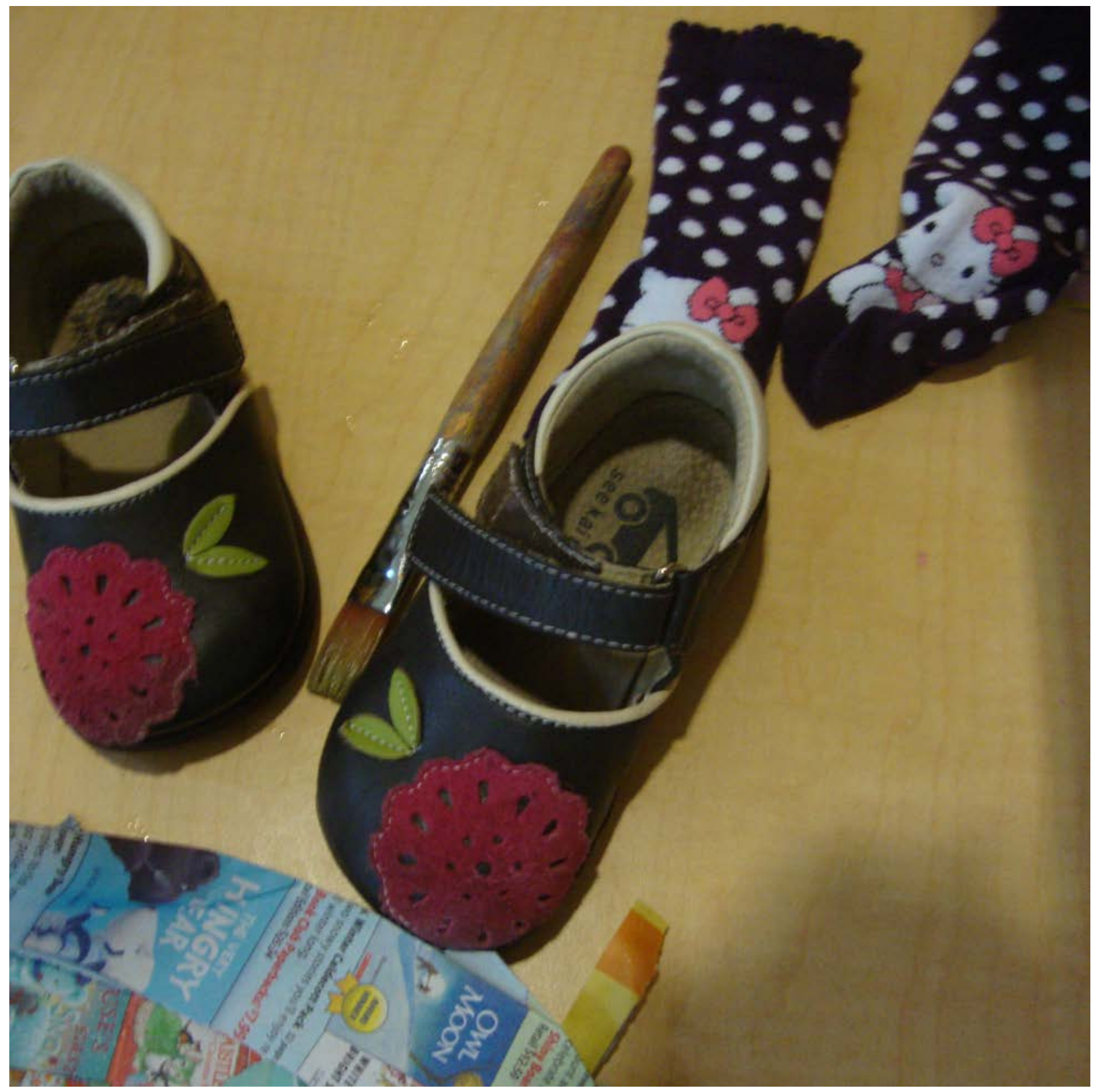


International Journal of Child, Youth, and Family Studies (2014): 5(4.2) 847-853

Figure 1. Traces left behind (Photo by Kelsey Wapenaar).

Many children have a strong desire to live out their days in bare feet; abandoned boots, socks, and shoes can be found throughout the classroom and the playground. Bare feet and materials come together like deeply engrained rituals. There is a rhythm to little feet and they leave behind traces. The sound of little feet echo throughout the room as an ever-present rhythmic cadence.

Again, this has also become a defining quality of the Dogwood Room where we observe the fierce struggle to remove a shoe and sock and free one's foot to intermingle with the elements of nature or the invitation of a large piece of clay. As educators, we can neither ignore nor curtail this strong desire to simply be shoe-less or sock-less and we begin to closely pay attention to the relationship between feet-earth-texture. By intentionally turning our attention to the space inbetween material and body, it becomes possible to observe the intra-activity of things as they meet and move together. Intra-activity speaks to a sense of embodiedness with the world; we begin to interpret our environment by penetrating the in-between spaces and becoming a part of it. Hillevi Lenz-Taguchi (2011) describes this further: "The flow of events thus becomes a collective and collaborative responsibility on behalf of all organisms present, whether they are human or non-human. Responsibility is thus built into the immanent relationship in-between everything in the encounters” (p. 48).

We are interested in what happens in the in-between places. We see the child not existing as separate from their environment, but rather as deeply entwined as body-touch-material are constantly at play. The objects and textures surrounding us in our environment are powerful and dynamic forces and we are in-context, overlapped, and mutually engaged. 


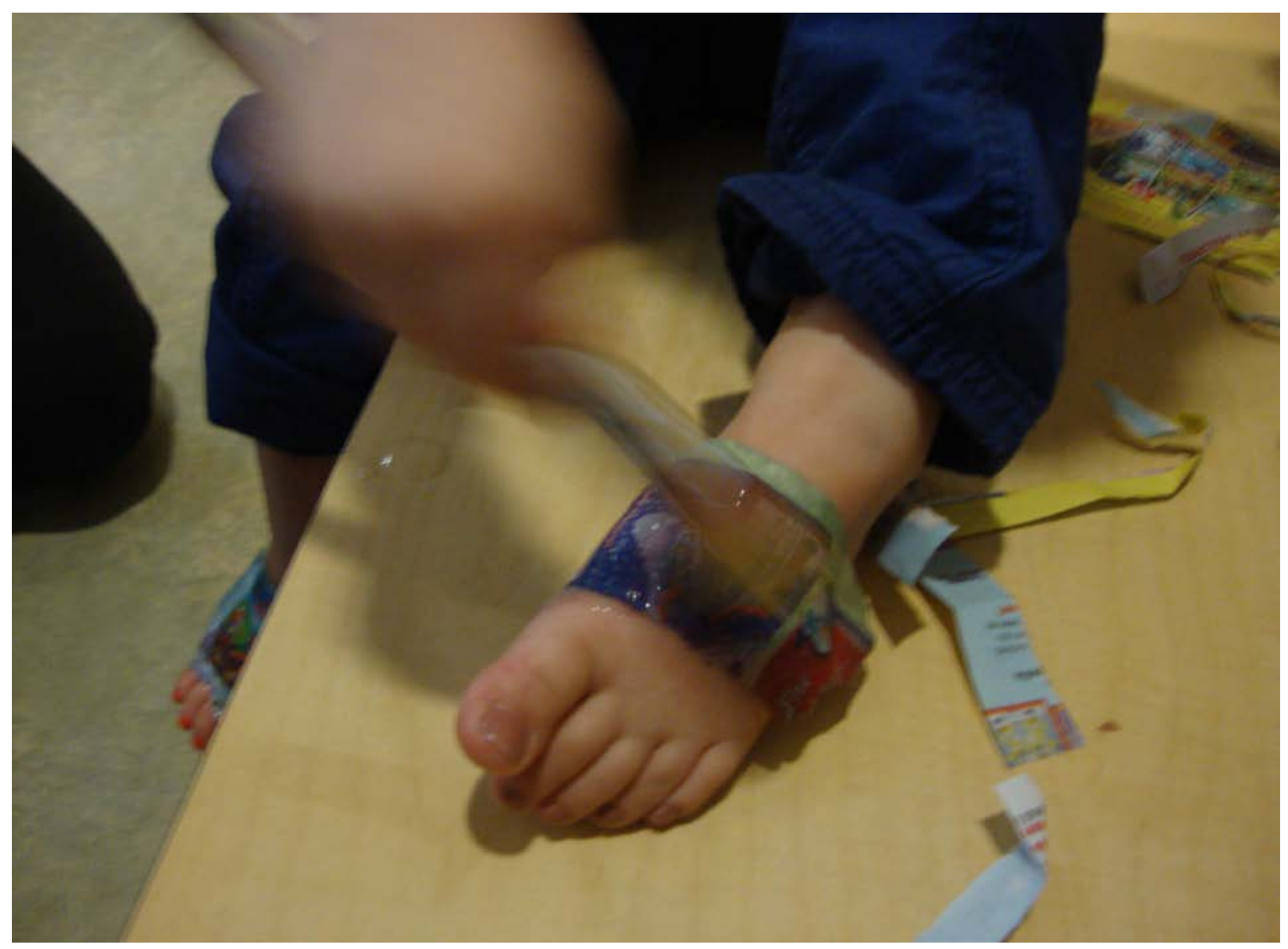

Figure 2. Paying attention to the in-between places (Photo by Kelsey Wapenaar).

\section{The Importance of Boots: Objects That Hold Meaning}

Footwear, but especially rubber boots, hold significant meaning within the culture of the Dogwood Room. The children have an interesting relationship with boots. They love to try them on, take them off, pile them together. Some boots become highly coveted while others seem less attractive. Anyone who spends time in our room will discover that footwear does not always have the purpose of keeping feet dry and protected. The adults in the room always seem to be on the outskirts of this peculiar and seemingly secret relationship between toddlers and boots, as we constantly try to regroup the boots together, in an orderly fashion, with the rightful owner. As a form of investigation, a pair of size 12 men's black rain boots were brought into the room as a way to explore and extract meanings on the value, importance, and attractiveness of boots. What is it about boots? 


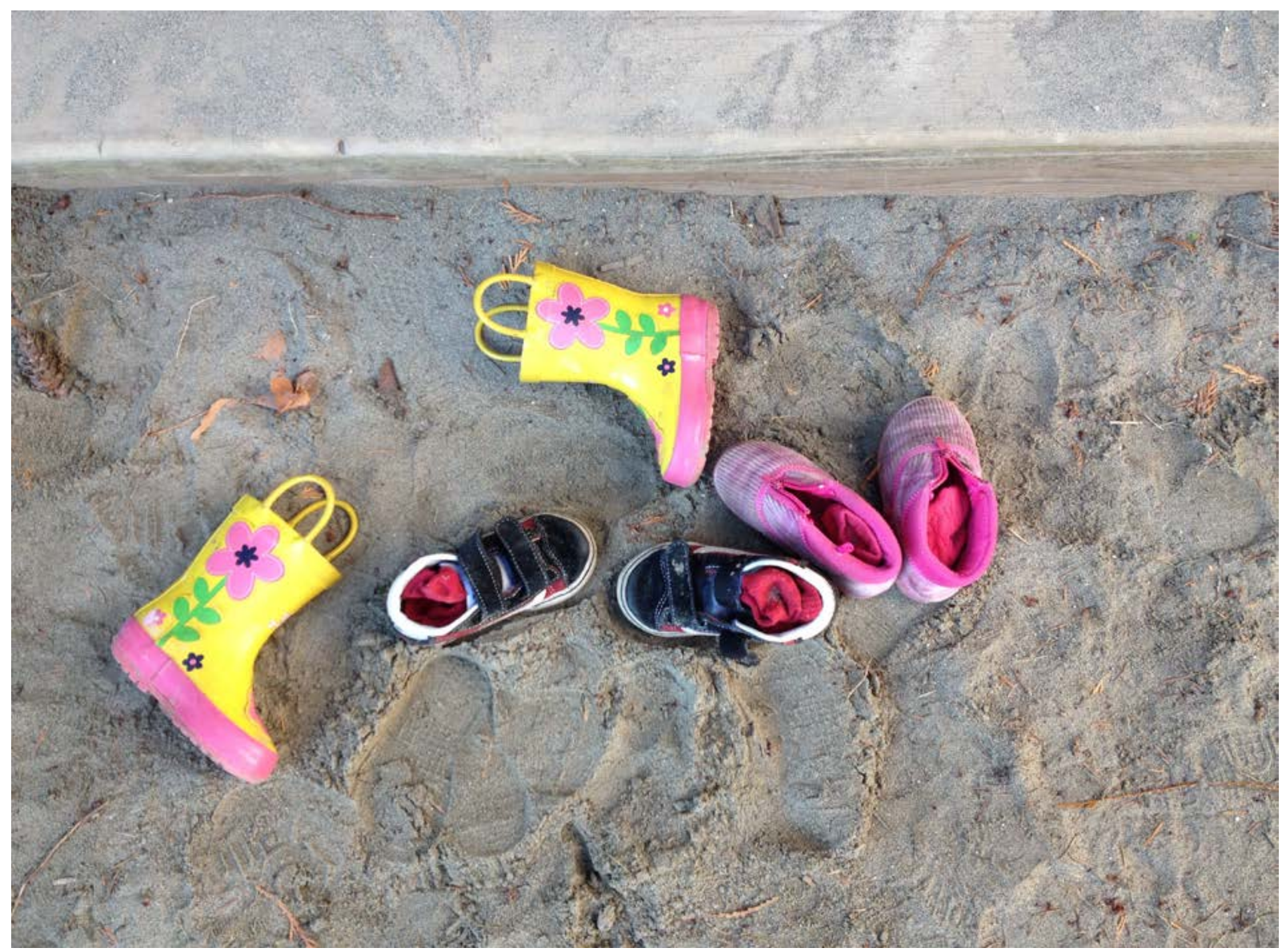

Figure 3. Collective rituals (Photo by the author).

These boots were quickly swept up in the movements of our room. They have traveled and come into contact with blocks, natural materials, shadows, paint, and much more. They eventually came into contact with papier mâché. The familiarity of boots has suddenly become unfamiliar and it seems as if our relationships with the boots have actually ruptured the assumed function of boots. The very purpose of boots has faded away and they seem to take on a symbolic characteristic, something that holds meaning and defines a certain characteristic of the collective culture of our room. We are suddenly challenged to re-think these everyday, somewhat normal and mundane objects as objects that hold identity, creative potential, even aesthetic qualities.

\section{Concealment and Revealment: Papier Mâché Encounters}

The act of covering up and peeling back layers to reveal something underneath is an everpresent ritual in the Dogwood Room. We decide to explore this idea further through papiermâché on every day familiar objects such as boots, a large orange pylon, and a plastic bike from the playground. The paper we are using holds deep curiosity and meaning for the children as we are recycling and tearing Scholastic book orders, which the children have always taken great delight in reading. Each shred of paper holds an image of their beloved characters (Dora, Max from Where The Wild Things Are, Thomas The Tank Engine, and so on). This process comes together as an assemblage of bodies-iconic images-paper-objects-space-time. These forces become entwined and layered and they meet not as a singular event or idea, but rather, as an encounter. We 
notice a fascinating flow to this encounter, as paper is continuously layered and images and print disappear, only to reappear again as the layers are peeled back. It would seem as if there is no end to the process. Each element of this assemblage carries a unique voice or characteristic: They appear to be fluid, emergent and perhaps inter-dependent. There is no dominant voice, but rather, a flow of exchanges that move with varied intensities and rhythms.

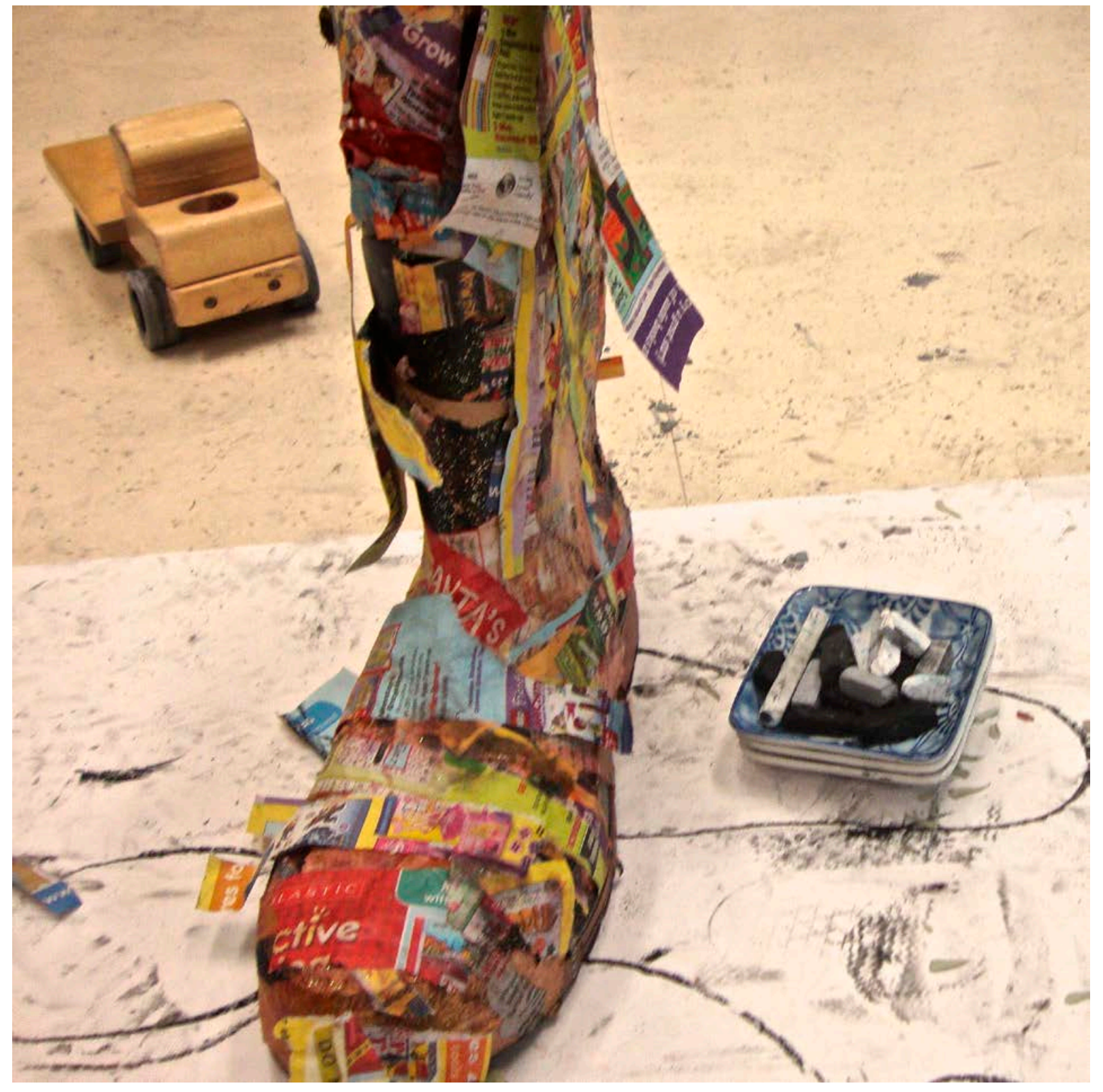

Figure 4. The inter-mingling of objects, material, space and time (Photo by Kelsey Wapenaar). 
International Journal of Child, Youth, and Family Studies (2014): 5(4.2) 847-853

Acknowledging the tensions, complexities, and rituals that emerge in a collective space allows us, as educators, to dig deep within the culture of our classrooms. Through this process, we traverse beyond the familiar and begin to look between subjects to acknowledge the beauty and rhythm of ritual and the shaping and shifting that occurs when materials, humans, objects, and time become entwined. The inter-connectedness of bodily gestures, the movements of materials, and the expressive power of objects elicits a deep understanding that within children's spaces, curriculum is an embodied experience, and it is embedded within everyday objects that hold distinctive meaning for children.

\section{Reference}

Lenz-Taguchi, H. (2011). Investigating learning. Participation and becoming in early childhood practices with a relational material approach. Global Studies of Childhood, 1(1), 39-48. http://dx.doi.org/10.2304/gsch.2011.1.1.36 